\title{
Grain Analysis of Atomic Force Microscopy Images via Topological Data Analysis
}

\section{Ali Nabi Duman}

King Fahd University of Petroleum and Minerals, Dhahran, Ash Sharqiyah, Saudi Arabia

Atomic force microscopy (AFM) is preferred in many applications due its ability to produce high resolution 3D images of almost any samples such as ceramic materials, metallic nanoparticles, flexible polymers, human cells etc. [3]. In order to utilise the unique properties of AFM data, it is important to conduct precise quantitative analysis.

One of the unique features of AFM data is its high vertical resolution which is vital to derive meaningful conclusions from the atomic and molecular structure of the samples [1]. This makes it possible to precisely determine the shape, size, and number of grains and nanoparticles which are related to the properties of the nanometric structures $[10,14]$. AFM data is usually processed prior to particle analysis. This procedure is often carried out manually for simplicity [8], but this makes it open to human bias and it has high processing time for a big number of particles.

The conventional methods, such as the threshold and watershed algorithms embedded to the softwares (e.g. WSxM [6] and Gwyddion [9]), might not be accurate and robust enough for grains and nanoparticles studies [13]. Threshold algorithms requires setting a global height threshold to isolate particles on a welllevelled background. Hence, particle height depends on the height threshold parameter, and it is not robust for non-constant background.

The images with non-constant background need to be preprocessed before the quantitative analysis. If not done correctly, the preprocessing called "levelling" might cause artefacts that might change the observed height of the grains, roughness values [15] and image size [12]. For example, the tilted image obtained via a method like the least-mean-squares fitting may not be entirely consistent with the real sample. The difference could be large, depending on the tilting angle [5]. A direct application of polynomial levelling may also be unsuccessful because the height of the raised background overlaps with the height of the lowered particles. This causes leaking of the background into the interested particles after thresholding [2]. Build-in software functions such as RMS Factor Threshold in SPIP image processing software to determine threshold depends on manually set parameters to get the optimal result. Hence, accurate levelling and threshold detection might be very time consuming in the case of large number of particles; and it is not uncommon to see poorly processed images in the literature [3].

Even though watershed algorithm based on the flow of water into basins gives better results in segmenting the grains, measuring grain heights after segmentation is not suitable as watershed may overflow the boundaries of grains. It is also not sensitive enough to detect the hierarchical structures, which are crucial in cases like multimeric proteins and hydrophobic surfaces [11].

Surface roughness parameters are also commonly used in the literature to quantitatively describe the granular surfaces. Even though there are many roughness parameters, root mean square roughness (RMS) is preferred in majority of the studies due its simplicity to calculate and interpret. However, RMS as a single number results in uncertainty in characterization of different surfaces. As demonstrated in [16], different surface topographies can give the same RMS. Additionally, RMS does not offer any spatial surface structure information and cannot make a difference between peaks and valleys which is crucial in granular surface analysis [7]. While the combination of existing roughness parameters are also proposed for characterization [4], it is necessary to develop robust methods that summarize statistical information about the peaks and valleys independent of the sampling area.

In this study, the main topological tool that we are using is persistent homology, which tracks how topological features appear and disappear in a nested sequence of topological spaces [17-20]. There are two main strengths of our approach: 
- It represents surfaces based on their topology making it invariant to small changes in shapes and hence robust to noise;

- It determines a range of coarse to fine scales of topological changes, thereby, summarizing large and small scale objects. In our case, it enables us to demonstrate micro and nanostructures of the surface in the same statistical summary without a need of thresholding or smoothing algorithms.

A wealth of geometric information can be extracted from a surface by the approach providing a more holistic analysis than the information from standard invariants such as surface roughness.

The persistent homological framework enables us to analyze complex and high dimensional data in a consistent manner [21-23]. It is robust to perturbations, independent of dimensions and scale, and provides a compact representation of the outputs. It lets us construct informative summaries of the shape of data. It can be applied to analyze both 2D and 3D images. The output of the persistent homology can be summarized visually using a persistence diagram, which is a collection of points in the plane where each point (x, y) characterizes a topological feature (e.g., hills, pores, cavities, etc.) that appears at height $\mathrm{x}$ and disappears at height $\mathrm{y}$. We say the feature has a persistence value (or life-span) of $\mathrm{y}-\mathrm{x}$. The features with low persistence can be considered as noise.

Our method is demonstrated in two types of AFM images: (i) $\mathrm{ZnO}$ and $\mathrm{Zn} 2 \mathrm{O} 3$ thin films grown by RFmagnetron sputtering [25-26]; and (ii) Polycarbonate wafers textured through crystallization in an acetone solution [24].

In this study, we suggest to use a topological data analysis method called persistent homology to avoid above mentioned limitations and provide a robust quantitative rules for grain analysis from unprocessed AFM images. Our method determines number particles and average height by avoiding the artefacts of preprocessing. It is also an accurate way of determining the roughness of the surface.

\section{References}

1. T. Arai et al. Scientific Reports, 7(1):4054, 2017.

2. S. D. Connell, et al. Quantitative Analysis of Structure and Dynamics in AFM Images of Lipid Membranes, chapter 3, pages 29-44. Springer New York, New York, NY, 2019.

3. P. Eaton and P. West. Atomic Force Microscopy. Oxford University Press, Inc., 2010.

4. M. Etxeberria, et al. Scanning, 37(6):429-437, 2019/12/08 2015.

5. J. Fu et al. Scanning, 30(1):41-46, 2019/11/26 2008.

6. I. Horcas, et al. Review of Scientific Instruments, 78(1):013705, 2019/11/25 2007.

7. R. Hristu, et al. 75(7):921-927, 2019/12/08 2012.

8. B. P. Marsh, et al. .Scientific Reports, 8(1):978, 2018.

9. D. Necas and P. Klapetek. Central European Journal of Physics, 10(1):181-188, 2012.

10. C. Y. Ngo, et al. Physical Review B, 74(24):245331-, 122006.

11. A. Owais, et al. Journal of Applied Polymer Science, 133(14), 2019/11/26 2016.

12. P. J. Ramon-Torregrosa, et al. Colloids and Surfaces A: Physicochemical and Engineering Aspects, 323(1):83- 93, 2008.

13. J. E. Ruiz, et al. Ultramicroscopy, 184:234-241, 2018.

14. N Sebaihi et al 2017 Meas. Sci. Technol. 28034006

15. J. Smith, et al. Transactions of the Institute of Metal Finishing, 81:B55-B58, 052003.

16. A. Szyszka, et al. Ultramicroscopy, 170:77-85, 2016.

17. P. Bendich, et al. IEEE Trans. Vis. Comput. Graph. 2010, 16, 1251-1260.

18. O. Delgado-Friedrichs, et al. IEEETrans. PatternAnal.Mach.Intell.2015,37,654-666. 
19. H. Kurtuldu et al.J.FluidMech.2011,682,543-557.

20. M. Carriere et al. Proceedings of the Computer Graphics Forum, Graz, Austria, 6-8 July 2015; Volume 34, pp. 1-12.

21. H. Edelsbrunner et al. Persistent homology. American Mathematical Society: Providence, RI, USA, 2008, Volume: 453, pp. 257-282.

22. H. Edelsbrunner et al. Proc. of the European Congress of Mathematics, Krakow, Poland, 2-7 July 2012.

23. R. Ghrist, Barcodes: The Persistent Topology of Data. Bul. Am. Math. Soc. 2008, 45, 61-75.

24. A. N. Duman et al. Coatings 2017, 7(9), 139.

25. B. Haider. Acta Physica Polonica A, 132:1325-1328, 102017.

26. B. Haider. Nanoscale Research Letters, 12(1):5, 2017. 